ALLEX JARDIM DA FonsECA ${ }^{1}$

Anderson Cesar Dalla-Benetta ${ }^{2}$

LEONARDO PIRES FERREIRA ${ }^{2}$

Cibelu Roldan NavarRo Martins ${ }^{3}$

CyNTHIA DANTAS MACEDO Lins ${ }^{4}$

\section{Quimioterapia neoadjuvante seguida de cirurgia radical em paciente grávida com câncer de colo de útero: relato de caso e revisão de literatura}

\author{
Neoadjuvant chemotherapy followed by radical surgery in pregnant \\ patient with invasive cervical cancer: case report and literature review
}

Palavras-chave

Neoplasias do colo do útero/cirurgia Neoplasias do colo do útero/quimioterapia Complicações neoplásicas na gravidez Terapia neoadjuvante Relatos de casos

Keywords

Uterine cervical neoplasms/surgery Uterine cervical neoplasms/drug therapy

Pregnancy complications, neoplastic Neoadjuvant therapy Case reports

\section{Resumo}

Em pacientes grávidas portadoras de câncer de colo de útero (CCU), as opções terapêuticas dependem da idade gestacional, do estágio clínico e do desejo da paciente. Alguns autores relataram casos de quimioterapia neoadjuvante seguidos de cirurgia radical nessas pacientes. O objetivo deste artigo foi revisitar o assunto, adicionar um novo caso e revisar a literatura. Relatamos o caso de uma mulher de 30 anos, na 24ª semana de gestação, que teve diagnóstico de câncer de colo de útero (carcinoma escamoso grau II), estágio IIB (Federação Internacional de Ginecologia e Obstetrícia - FIGO). Nulípara, a paciente recusou a interrupção da gravidez. Após meticuloso esclarecimento, a paciente aceitou tratamento com quimioterapia neoadjuvante com cisplatina $75 \mathrm{mg} / \mathrm{m}^{2}$ e vincristina $1 \mathrm{mg} / \mathrm{m}^{2}$, além de posterior avaliação de cirurgia radical e parto cirúrgico concomitantes. Quatros ciclos completos de quimioterapia foram administrados sem atrasos ou efeitos adversos importantes. Poucos dias antes da data programada para a cirurgia, a paciente foi admitida em trabalho de parto na $37^{a}$ semana de gestação. Devido à resposta clínica completa do tumor, a equipe obstétrica optou por monitorar o trabalho de parto, e a paciente deu à luz um recém-nascido de $2.450 \mathrm{~g}$, sem intercorrências. A cirurgia radical foi realizada três dias após o parto, e a análise histopatológica revelou carcinoma confinado ao colo sem envolvimento linfonodal. Mãe e filho se encontram em bom estado geral 12 meses após o parto. Quimioterapia baseada em cisplatina durante o segundo ou terceiro trimestre da gravidez parece ser uma opção para as pacientes que não desejam a interrupção da gravidez enquanto se aguarda a maturidade fetal. Entretanto, estudos adicionais são necessários para confirmar o prognóstico e a segurança dos recém-nascidos e das pacientes.

\section{Abstract}

The treatment options for pregnant patients with invasive cervical cancer (ICC) depend on gestational age, clinical stage and the patient's wishes. Some authors have reported cases of neoadjuvant chemotherapy followed by radical surgery in these patients. The aim of this paper was to revisit this subject and to add a new case and review the literature. We report the case of a 30 year-old woman in the $24^{\text {th }}$ week of gestation. She was diagnosed with ICC (squamous cell carcinoma grade 2), stage IIB (International Federation of Gynecology and Obstetrics - FIGO). Nulliparous, the patient refused to interrupt the pregnancy. After meticulous counseling, the patient accepted treatment with neoadjuvant chemotherapy (cisplatin $75 \mathrm{mg} / \mathrm{m}^{2}$ and vincristine $1 \mathrm{mg} / \mathrm{m}^{2}$ ) and subsequent evaluation of radical surgery concomitant to a cesarean section. Four complete cycles of chemotherapy were administered without delays or significant adverse effects. A few days before the date scheduled for surgery, the patient was admitted in advanced labor (37'th week of gestation). Due to tumor clinical response, the obstetric team decided to monitor the labor, and the patient gave birth to a male newborn $(2,450 \mathrm{~g})$ uneventfully. Radical surgery was performed three days after birth, and histopathology analysis revealed carcinoma confined to the cervix without lymphatic involvement. Mother and son are in good general condition 12 months after delivery. Cisplatin-based chemotherapy during the second or third trimester of pregnancy appears to be a safe option for patients who do not wish to interrupt a pregnancy while awaiting fetal maturity. However, additional studies are needed to confirm the prognosis and assure the safety of newborns and patients.
Correspondência:

Allex Jardim da Fonseco Unidade de Alta Complexidade em Oncologia do Hospital Geral de Roraima - Boa Vista (RR), Brasil Avenida Brigadeiro Eduardo Gomes, $\mathrm{s} / \mathrm{n}$ CEP 69307.755 - Boa Vista (RR), Brasi E-mail: allex.jardim@bol.com.br
Unidade de Alta Complexidade em Oncologia do Hospital Geral de Roraima - Boa Vista (RR), Brasil.

Oncologista Clínico do Serviço de Oncologia do Hospital Geral de Roraima - Boa Vista (RR), Brasil; Mestre em Ciências Econômicas pela Universidade Federal do Rio Grande do Sul - UFRGS - Porto Alegre (RS), Brasil.

${ }^{2}$ Cirurgião Oncológico do Serviço de Oncologia do Hospital Geral de Roraima - Boa Vista (RR), Brasil.

${ }^{3}$ Hematologista e Coordenadora do Serviço de Oncologia do Hospital Geral de Roraima - Boa Vista (RR), Brasil.

${ }^{4}$ Ginecologista e Obstetra do Hospital Materno Infantil de Roraima - Boa Vista (RR), Brasil.

Conflito de interesses: não há

Os autores declaram não ter havido suporte financeiro ou de qualquer outra natureza para a elaboração deste manuscrito. 


\section{Introdução}

Aproximadamente 2\% dos casos de câncer de colo de útero (CCU) ocorrem em mulheres grávidas. Metade desses casos é descoberta no puerpério; $30 \%$ durante o parto; e apenas $20 \%$ no período pré-natal ${ }^{1}$. Isso torna o CCU uma das malignidades mais comuns durante a gestação (assim como o câncer de mama), com incidência estimada de aproximadamente 1 caso por 1.000 a 2.500 nascidos vivos ${ }^{2-4}$.

Mais comumente, o CCU se manifesta em estádios precoces durante a gravidez. Em estudo que revisou 494 casos de CCU em gestantes, 83,9\% dos casos foram estadiados como I e 11,8\% como II (Federação Internacional

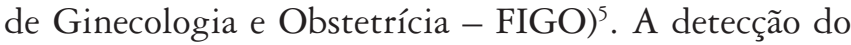
câncer em estágios iniciais é três vezes mais frequente em mulheres grávidas quando comparadas às não grávidas, provavelmente devido ao rastreio citológico pré-natal rotineiro e à dificuldade que mulheres com câncer localmente avançado podem enfrentar para engravidar.

Apesar de estudos retrospectivos não revelarem diferença no prognóstico de pacientes com CCU grávidas em comparação às não grávidas, os dados são limitados, especialmente quanto ao seguimento de longo prazo ${ }^{6}$. Alguns autores têm publicado resultados de atrasos deliberados para o tratamento radical (cirurgia com ou sem tratamento adjuvante e radioquimioterapia) com o intuito de se alcançar a maturidade fetal ${ }^{7-10}$. A maioria dos relatos de casos consiste de pacientes com lesão no estádio I, com média de atraso programado para o tratamento de 12 semanas (1 a 32 semanas). Na maioria dos casos relatados, observou-se alguma progressão tumoral durante o atraso, porém, com prognóstico materno-fetal satisfatório $^{7-10}$. Entretanto, raros casos dessa abordagem foram relatados em pacientes com doença localmente avançada, além da recomendação de se evitar o atraso no tratamento para pacientes com tumor maior que $4 \mathrm{~cm}$ ou com linfonodos positivos ${ }^{10}$. Nesses casos, a avaliação linfonodal pode ser realizada por via laparoscópica ${ }^{11}$.

A dificuldade de fazer predições sobre o prognóstico gera incertezas para pacientes e médicos. Nesse sentido, o manejo dessas pacientes ainda é um desafio para as equipes médicas em virtude da inexistência de estudos randomizados abordando o assunto. Em geral, as condutas são baseadas em evidências de estudos de mulheres não grávidas. O objetivo deste trabalho foi rever as estratégias de tratamento de pacientes grávidas portadoras de CCU, especialmente o papel da quimioterapia neoadjuvante para controlar a doença neoplásica enquanto se aguarda a maturidade fetal, assim como sua segurança para a paciente e para o concepto, além de adicionar um novo caso à literatura mundial.

\section{Relato de caso}

Mulher negra de 30 anos, grávida de 24 semanas, foi encaminhada ao serviço devido a sangramento vaginal. O exame especular evidenciou lesão tumoral infiltrando o colo de útero superiormente, friável e sangrante, e o exame físico revelou que a massa acometia parcialmente o paramétrio bilateralmente. O fundo do útero era palpável a $22 \mathrm{~cm}$ do púbis, e os batimentos cardíacos fetais eram de $120 \mathrm{bpm}$. Uma biópsia cervical foi realizada revelando carcinoma de células escamosas moderadamente diferenciado. O exame de ressonância magnética do abdômen total revelou lesão cervical de $3 \times 4 \mathrm{~cm}$ acometendo o paramétrio bilateralmente, sem sinais de dilatação de vias urinárias, lesões a distância ou linfonodomegalia locorregional. Cistoscopia e retosigmoidoscopoia descartaram invasão de bexiga e reto. A radiografia de tórax foi normal. O câncer da paciente foi estadiado como IIB FIGO. A paciente era nulípara e rejeitou a interrupção da gravidez. Após um meticuloso esclarecimento das opções e dos riscos relacionados, a paciente, juntamente com a equipe médica, decidiu iniciar quimioterapia neoadjuvante com cisplatina $75 \mathrm{mg} / \mathrm{m}^{2}$ e vincristina $1 \mathrm{mg} / \mathrm{m}^{2}$ a cada 21 dias, com programação de parto cirúrgico concomitante à cirurgia radical. Os exames laboratoriais pré e pós-tratamento estão ilustrados na Tabela 1.

Quatro ciclos de quimioterapia foram administrados nas semanas 25, 28, 31 e 34 . O tratamento foi bem tolerado, sem toxicidades relevantes ou atrasos. Após o primeiro ciclo, a paciente relatou cessação do sangramento vaginal e das dores pélvicas. $\mathrm{Na} 28^{\mathrm{a}}$ semana, a paciente foi submetida à ultrassonografia obstétrica com dopplerfluxometria,

Tabela 1 - Resultados de exames laboratorias pré e pós-tratamento

\begin{tabular}{|c|c|c|c|c|c|}
\hline Exames laboratoriais & $\begin{array}{l}\text { IG } 24^{\mathrm{a}} \text { semana } \\
\text { (pré QT) }\end{array}$ & $\begin{array}{l}\text { IG } 28^{\circ} \text { semana } \\
\text { ( } 2^{\circ} \text { ciclo QT) }\end{array}$ & $\begin{array}{l}\text { IG } 31^{\mathrm{a}} \text { semana } \\
\text { (3 } 3^{\circ} \text { ciclo QT) }\end{array}$ & $\begin{array}{l}\text { IG } 34^{\circ} \text { semana } \\
\text { (4 } 4^{\circ} \text { ciclo QT) }\end{array}$ & Pós-operatório imediato \\
\hline Hemoglobina (g/L) & 11,5 & 11,3 & 10,5 & 10,6 & 9,3 \\
\hline Leucometria $\left(\mathrm{n} / \mathrm{mm}^{3}\right)$ & 7.430 & 5.320 & 4.300 & 5.780 & 12.430 \\
\hline Contagem de plaquetas $\left(\mathrm{n} / \mathrm{mm}^{3}\right)$ & 345.000 & 256.000 & 155.000 & 230.000 & 252.000 \\
\hline Creatinina (mg/dL) & 0,8 & 0,9 & 1,0 & 0,9 & 1,1 \\
\hline
\end{tabular}

IG: idade gestacional; QT: quimioterapia; PO: pós-operatório. 


\begin{tabular}{|c|c|c|c|c|c|c|c|c|c|c|c|c|c|c|c|c|c|c|}
\hline 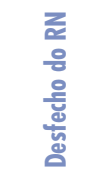 & $\begin{array}{l}\text { D } \\
\text { 号 } \\
\text { i } \\
\text { 言 } \\
\text { 产 }\end{array}$ & 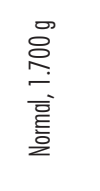 & 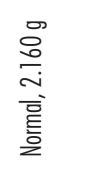 & 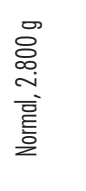 & 豆 & 豆 & 亨 & 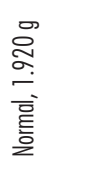 & 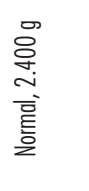 & 豆 & 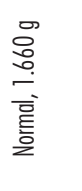 & 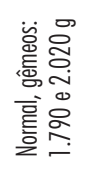 & 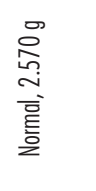 & $\begin{array}{l}\text { 宫 } \\
\text { ì } \\
\text { 言 } \\
\text { 究 }\end{array}$ & 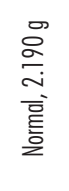 & & 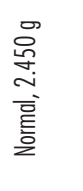 & 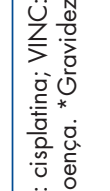 \\
\hline 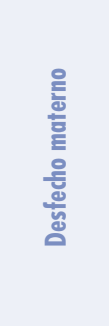 & 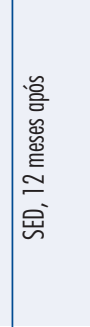 & $\begin{array}{l}\text { o. } \\
\text { 产 } \\
\text { 号 } \\
\text { 岕 }\end{array}$ & 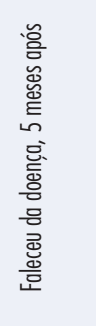 & 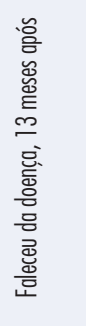 & 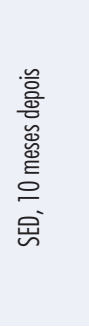 & 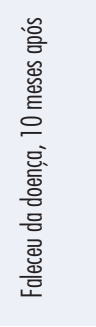 & 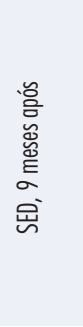 & 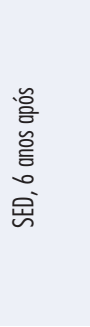 & 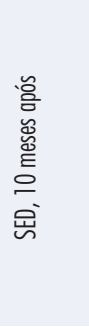 & 怘 & $\begin{array}{l}\text { 言 } \\
\text { 言 } \\
\text { वे } \\
\text { 点 }\end{array}$ & $\begin{array}{l}\text { 高 } \\
\text { 訔 } \\
\text { 㤩 }\end{array}$ & 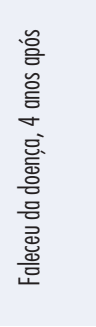 & $\begin{array}{l}\text { 言 } \\
\text { 产 } \\
\text { 足 } \\
\text { 品 }\end{array}$ & 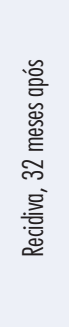 & 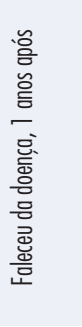 & 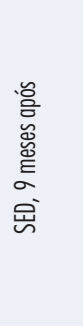 & 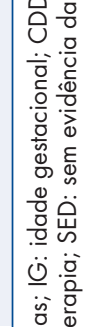 \\
\hline 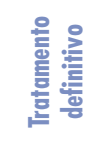 & $\dddot{x}$ & 足 & $\begin{array}{l}\stackrel{\underline{a}}{+} \\
\stackrel{+}{x}\end{array}$ & 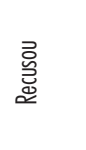 & 吕 & $\begin{array}{l}\frac{5}{a+} \\
+ \\
b\end{array}$ & $\begin{array}{l}\underline{\underline{a}} \\
+ \\
\underline{\underline{x}}\end{array}$ & $\begin{array}{l}\sigma \\
+ \\
\stackrel{+}{\mp}\end{array}$ & 足 & $\begin{array}{l}\text { ta } \\
+ \\
\stackrel{+}{0}\end{array}$ & $\begin{array}{l}\stackrel{5}{a} \\
\stackrel{+}{0}\end{array}$ & 足 & 足 & $\begin{array}{l}\vdots \\
+ \\
+ \\
\stackrel{+}{x}\end{array}$ & 王 & 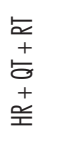 & $\dddot{x}$ & 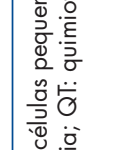 \\
\hline 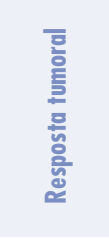 & 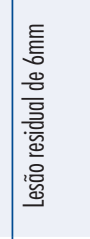 & 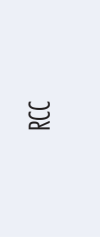 & 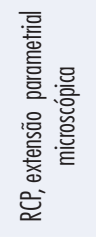 & ¿̀ & 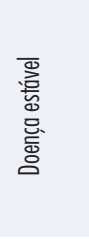 & 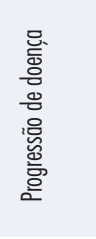 & 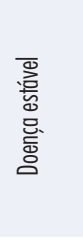 & 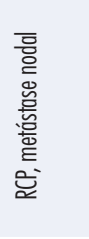 & Ё & ¿̀ & 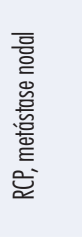 & 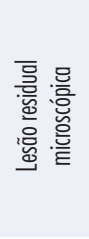 & 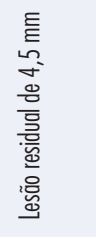 & 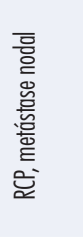 & 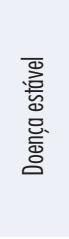 & $\begin{array}{l}\text { 営 } \\
\text { 总 } \\
\text { 总 }\end{array}$ & 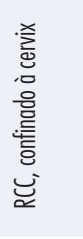 & 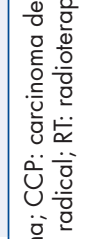 \\
\hline 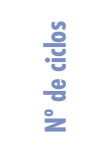 & 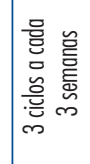 & 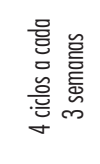 & 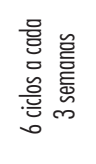 & 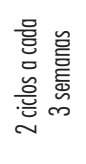 & 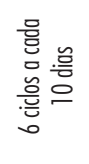 & 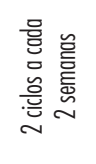 & 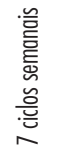 & 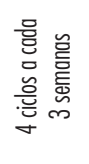 & 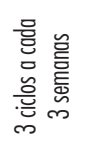 & 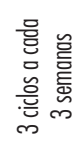 & 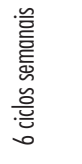 & 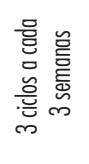 & 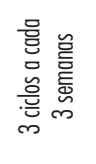 & 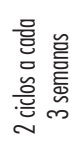 & 응 & 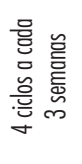 & 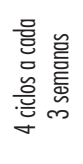 & 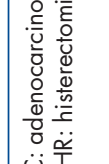 \\
\hline 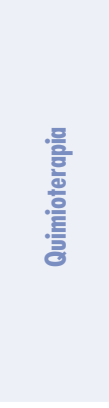 & 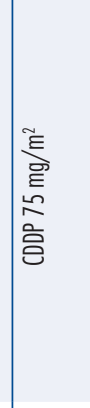 & 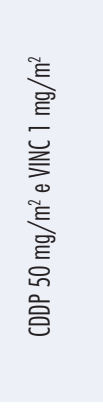 & 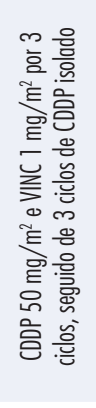 & 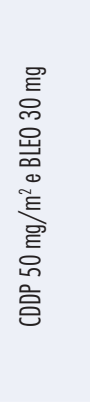 & 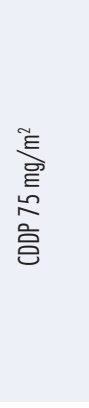 & 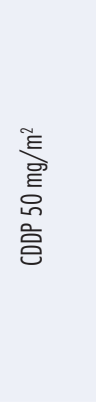 & 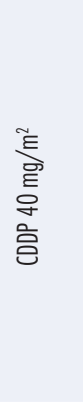 & 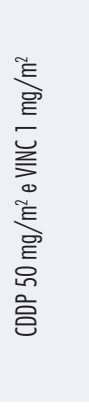 & 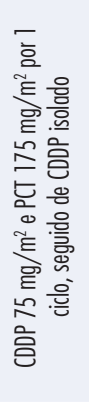 & 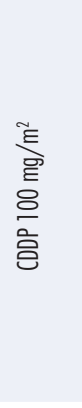 & 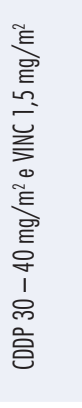 & $\begin{array}{l}\text { है } \\
\text { हू } \\
\text { Dे } \\
\text { 㐔 }\end{array}$ & 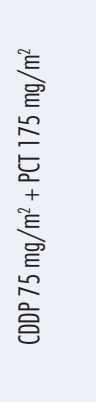 & & 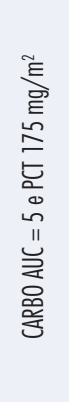 & 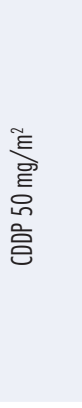 & 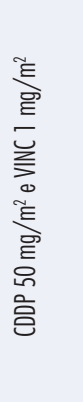 & 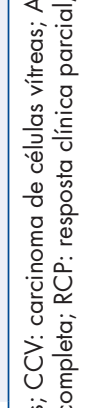 \\
\hline 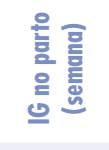 & $\approx$ & $\tilde{m}$ & 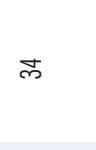 & $\infty$ & $\tilde{m}$ & $\stackrel{*}{\sim}$ & $\ddot{m}$ & $\ddot{m}$ & $\stackrel{m}{m}$ & m & $\bar{m}$ & $\approx$ & in & & $m_{m}$ & $\widetilde{m}$ & $\hat{m}$ & 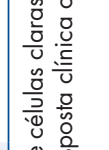 \\
\hline 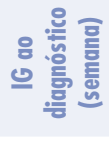 & $=$ & 尺 & $\simeq$ & $\Xi$ & $\stackrel{n}{2}$ & $\approx$ & $\mathscr{\sim}$ & $\stackrel{-}{0}$ & $\stackrel{\sigma}{=}$ & $\bar{\sim}$ & $\mathscr{\sim}$ & 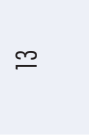 & $\stackrel{\sim}{\sim}$ & $\stackrel{\sim}{~}$ & $\stackrel{\infty}{\sim}$ & $\stackrel{n}{n}$ & 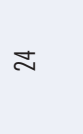 & 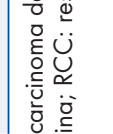 \\
\hline 음업 & $\Phi$ & $\begin{array}{l}\stackrel{\bar{z}}{\overline{\bar{z}}} \\
\underline{\underline{\underline{m}}}\end{array}$ & $\leqq$ & $\cong$ & $\Phi$ & $\stackrel{\varrho}{\underline{\underline{\underline{n}}}}$ & $\cong$ & $\leqq$ & $\cong$ & $\cong$ & $\stackrel{\varrho}{\underline{\underline{1}}}$ & $\Phi$ & $\Phi$ & $\cong$ & $\begin{array}{l}\text { 訔 } \\
\underline{\underline{\underline{\underline{a}}}}\end{array}$ & $\cong$ & $\cong$ & 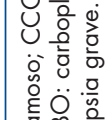 \\
\hline 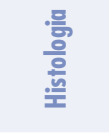 & ب & 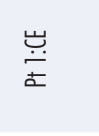 & 岕 & 岂 & U & ت & U & U & U & こ્પ & 己 & 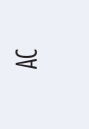 & 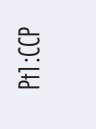 & $\begin{array}{l}\text { 㟧 } \\
\text { L }\end{array}$ & $\begin{array}{l}\text { 岕 } \\
\text { 足 }\end{array}$ & U & 岂 & 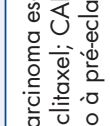 \\
\hline 호를 & 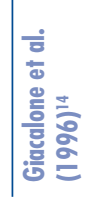 & 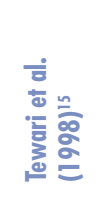 & & 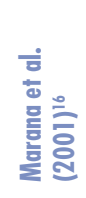 & 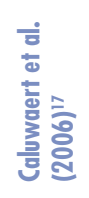 & 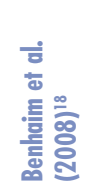 & 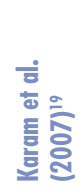 & 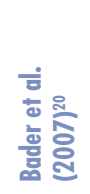 & 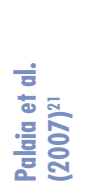 & 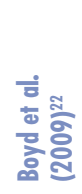 & 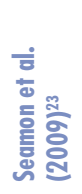 & 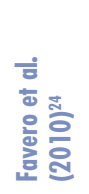 & 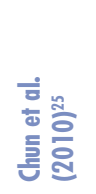 & & & 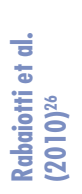 & 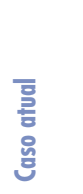 & 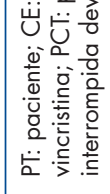 \\
\hline
\end{tabular}


com resultados normais. Não havia sinais de restrição de crescimento intrauterino, o índice de líquido amniótico era compatível com a idade gestacional e a placenta estava localizada na região fúndica. Após o terceiro ciclo, a avaliação ginecológica revelou resposta clínica completa.

A cirurgia foi programada para a $38^{a}$ semana de gestação. Três dias antes, na $37^{\mathrm{a}}$ semana +4 dias, a paciente foi admitida em trabalho de parto com $7 \mathrm{~cm}$ de dilatação do cérvix. A equipe de obstetrícia optou por monitorização fetal e prosseguimento do parto vaginal. Após três horas, a paciente deu à luz um recém-nascido do sexo masculino, sem intercorrências, com Índice Apgar de $1^{\circ}$ e $5^{\circ}$ minutos de 8 e 9 , respectivamente, pesando 2.450 g (abaixo do percentil 10 de peso fetal estimado para a idade gestacional). Não foi evidenciado sangramento anormal no puerpério. Três dias depois, a paciente foi submetida à histerectomia radical com linfadenectomia pélvica e paraaórtica, também sem intercorrências. A mãe e o recém-nascido receberam alta no quinto dia de pós-operatório, ambos em excelentes condições gerais. A análise histopatológica revelou carcinoma escamoso moderadamente diferenciado restrito ao colo uterino, com $11 \mathrm{~mm}$ de invasão e $20 \mathrm{~mm}$ de diâmetro. Os paramétrios e a cúpula vaginal foram livres de neoplasia. As margens cirúrgicas foram livres e não se evidenciou envolvimento linfonodal. Não foi indicado nenhum outro tratamento adjuvante. O seguimento da paciente realizou-se com consultas clínicas trimestrais no serviço de Oncologia, associado a exame especular e citológico vaginal, ultrassonografia abdominal total e radiografia de tórax. Após seguimento de 12 meses, a mãe não apresentou nenhuma evidência da doença neoplásica e a criança apresentou desenvolvimento normal.

\section{Discussão}

Em mulheres não grávidas, o uso de quimioterapia neoadjuvante para o CCU tem sido associado à conversão da doença localmente avançada e inoperável em doença operável, além de poder controlar micrometástases em sítios distantes, bem como em linfonodos locorregionais. Dois estudos fase III em pacientes não grávidas com CCU localmente avançado revelaram benefício de sobrevida global para o grupo tratado com quimioterapia neoadjuvante, seguida de histerectomia radical, quando comparado à cirurgia isolada, à radioterapia isolada e à quimioterapia combinada com a radioterapia ${ }^{12,13}$.

Encorajados por esses resultados, alguns autores relataram suas experiências com o uso de quimioterapia neoadjuvante para o tratamento de mulheres grávidas, portadoras de CCU, com o intuito de atingir regressão tumoral ou estabilização enquanto se aguarda a viabilidade fetal. Até a presente data, encontramos dados disponíveis de 16 casos na literatura mundial ${ }^{14-26}$.
A análise destes e do presente caso, encontra-se na Tabela 2. Observamos que 13 das 16 pacientes grávidas tratadas com quimioterapia neoadjuvante apresentavam neoplasia do tipo histológico, carcinoma de células escamosas $(76,4 \%)$, e foram estadiadas como I ou II FIGO ( $47 \%$ e $41,1 \%$, respectivamente). Em média, três ciclos de quimioterapia foram administrados durante a gravidez. A cisplatina foi o quimioterápico mais utilizado (16 casos), comumente associado à vincristina ou paclitaxel.

A quimioterapia neoadjuvante foi eficaz em todos os casos para permitir a evolução da gravidez até a viabilidade fetal, e não foram relatadas complicações graves nos recém-nascidos. Revelou-se também eficiente para reduzir ou estabilizar a neoplasia em todos os casos durante a gravidez, com exceção de um. $\mathrm{O}$ tratamento definitivo realizado em 12 das 17 pacientes (70,5\%) foi baseado em histerectomia radical concomitante ao parto cirúrgico, e em radioquimioterapia para três das pacientes $(17,6 \%)$. Uma delas recusou o tratamento definitivo, e a paciente do nosso caso foi tratada com histerectomia radical após parto normal. Onze das 17 pacientes $(64,7 \%)$ encontravam-se sem evidência da doença até o momento da publicação dos relatos. Uma paciente teve a gravidez interrompida na sexta semana de quimioterapia ( $28^{a}$ semana de gestação) devido à doença hipertensiva específica da gravidez (pré-eclâmpsia grave).

Estudos documentaram o risco teratogênico da quimioterapia isolada ( 7 a 17\%) ou em combinação (até 25\%) quando aplicada no primeiro trimestre da gestação ${ }^{27,28}$. Entretanto, o risco de complicações fetais ao nascimento quando a quimioterapia foi administrada no segundo ou terceiro semestre, revelou-se similar ao da população de grávidas em geral (1 a 2\%). O quimioterápico com maior atividade para o CCU é a cisplatina. Essa droga vem se mostrando segura quando administrada durante a gravidez, pois menos de um terço da dose atravessa a placenta. A concentração da cisplatina nos vasos do cordão umbilical corresponde a $30 \%$ da concentração da droga no soro materno, e no líquido amniótico corresponde a aproximadamente $10 \%{ }^{29}$. A neutropenia transitória no recém-nascido é uma reação adversa conhecida. Por isso, recomenda-se que a última aplicação de quimioterapia, preceda em pelo menos três semanas a data planejada do parto ${ }^{27}$.

Nossa paciente apresentou boa tolerância ao tratamento quimioterápico. De todos os casos relatados, apenas duas pacientes $(11,7 \%)$ apresentaram toxicidade limitante à quimioterapia (associação de cisplatina com vincristina e paclitaxel $)^{15,21}$. Nesses casos, os sintomas gastrointestinais e efeitos hematológicos foram controlados com a manutenção da cisplatina como tratamento isolado. Nesses dois casos, as pacientes foram tratadas com esquemas combinados de duas drogas antes da $20^{\mathrm{a}}$ semana de gestação. 
O peso do recém-nascido do presente caso estava abaixo do percentil 10, segundo estimativa de peso fetal de Hadlock (2.513 g para a idade gestacional) ${ }^{30}$. Não foi encontrada outra causa para o baixo peso ao nascer em nosso caso clínico senão, o tratamento com quimioterapia. Essa complicação esteve presente, em algum grau, em quase todos os casos relatados na literatura (Tabela 2). As complicações fetais mais comumente relacionadas ao tratamento quimioterápico durante o segundo e o terceiro trimestres de gestação são a restrição do crescimento intrauterino, prematuridade e baixo peso ao nascer ${ }^{28}$.

O parto vaginal normalmente não é recomendado em pacientes portadoras de CCU, pois a dilatação cervical pode liberar células tumorais viáveis que podem se implantar ao longo do canal do parto. A episiotomia deve ser evitada devido ao risco de implantação tumoral no sítio da incisão ${ }^{31}$. Pelo menos 16 casos de implantação tumoral na cicatriz da episiotomia (em pacientes que não receberam quimioterapia neoadjuvante) foram relatados na literatura, todos afetando o prognóstico da paciente. Metade das pacientes que apresentaram recorrência do CCU no sítio da episiotomia morreu da doença ${ }^{5}$. Não encontramos outros casos relatados de quimioterapia neoadjuvante para colo de útero precedendo parto normal. Assim, prevalece a recomendação de programação de parto cirúrgico para todas as gestantes portadoras de CCU, submetidas ou não à quimioterapia neoadjuvante. Em nossa paciente, a opção de monitorizar o trabalho de parto normal não foi programada, mas aconteceu devido ao bom controle da doença com a quimioterapia e, sobretudo, à admissão da paciente já com dilatação avançada do colo uterino, segundo julgamento da equipe obstétrica.

Ressaltamos que um importante aspecto ético é o extensivo esclarecimento da paciente sobre os riscos e benefícios da estratégia envolvendo a quimioterapia neoadjuvante. Essa estratégia não é baseada em resultados de estudos randomizados, mas em analogia a estudos realizados em portadoras de CCU não grávidas.

Concluímos que a quimioterapia baseada em cisplatina durante o segundo ou terceiro trimestre da gravidez parece ser uma opção segura para pacientes grávidas portadoras de CCU que não desejam a interrupção da gravidez enquanto se posterga o tratamento definitivo com o intuito de alcançar a maturidade fetal. Entretanto, ensaios randomizados e estudos com seguimentos mais prolongados são necessários para confirmar o desfecho seguro dos recém-nascidos e, sobretudo, das pacientes.

\section{Declaração dos autores}

Os autores declaram não haver conflito de interesses, suporte financeiro ou de qualquer outra natureza para a elaboração deste manuscrito. A paciente referida foi esclarecida e consentiu formalmente com este relato de caso.

\section{Referências}

1. Smith LH, Dalrymple JL, Leiserowitz GS, Danielsen B, Gilbert WM. Obstetrical deliveries associated with maternal malignancy in California, 1992 through 1997. Am J Obstet Gynecol. 2001;184(7):1504-12.

2. Creasman WT. Cancer and pregnancy. Ann N Y Acad Sci. 2001;943:281-6.

3. Nguyen C, Montz FJ, Bristow RE. Management of stage I cervical cancer in pregnancy. Obstet Gynecol Surv. 2000;55(10):633-43.

4. Smith LH, Danielsen B, Allen ME, Cress R. Cancer associated with obstetric delivery: results of linkage with the California cancer registry. Am J Obstet Gynecol. 2003;189(4): 1 128-35.

5. Van Calsteren K, Vergote I, Amant F. Cervical neoplasia during pregnancy: diagnosis, management and prognosis. Best Pract Res Clin Obstet Gynaecol. 2005;19(4):61 1-30.

6. Pettersson BF, Andersson S, Hellman K, Hellström AC. Invasive carcinoma of the uterine cervix associated with pregnancy: 90 years of experience. Cancer. 2010;1 16(10):2343-9.

7. Duggan B, Muderspach LI, Roman LD, Curtin JP, d'Ablaing G 3rd, Morrow CP. Cervical cancer in pregnancy: reporting on planned delay in therapy. Obstet Gynecol. 1993;82(4 Pt 1):598-602.

8. Sood AK, Sorosky JI, Krogman S, Anderson B, Benda J, Buller RE. Surgical management of cervical cancer complicating pregnancy: a case-control study. Gynecol Oncol. 1996;63(3):294-8.

9. Ishioka S, Ezaka Y, Endo T, Nagasawa K, Shimizu A, Sato A, et al. Outcomes of planned delivery delay in pregnant patients with invasive gynecologic cancer. Int J Clin Oncol. 2009;14(4):321-5.
10. Germann N, Haie-Meder C, Morice P, Lhomme C, Duvillard $P$, Hacene $K$, et al. Management and clinical outcomes of pregnant patients with invasive cervical cancer. Ann Oncol. 2005; 16(3):397-402.

11. Favero G, Chiantera V, Oleszczuk A, Gallotta V, Hertel H, Herrmann J, et al. Invasive cervical cancer during pregnancy: laparoscopic nodal evaluation before oncologic treatment delay. Gynecol Oncol. 2010;118(2):123-7.

12. Benedetti-Panici P, Greggi S, Colombo A, Amoroso M, Smaniotto $D$, Giannarelli $D$, et al. Neoadjuvant chemotherapy and radical surgery versus exclusive radiotherapy in locally advanced squamous cell cervical cancer: results from the Italian multicenter randomized study. J Clin Oncol. 2002;20(1):179-88.

13. Sardi JE, Giaroli A, Sananes C, Ferreira M, Soderini A, Bermudez $A$, et al. Long-term follow-up of the first randomized trial using neoadjuvant chemotherapy in stage $\mathrm{lb}$ squamous carcinoma of the cervix: the final results. Gynecol Oncol. 1997;67(1):61-9.

14. Giacalone PL, Laffargue F, Benos P, Rousseau O, Hedon B. Cisplatinum neoadjuvant chemotherapy in a pregnant woman with invasive carcinoma of the uterine cervix. Br J Obstet Gynaecol. 1996; 103(9):932-4.

15. Tewari K, Cappuccini F, Gambino A, Kohler MF, Pecorelli S, DiSaia PJ. Neoadjuvant chemotherapy in the treatment of locally advanced cervical carcinoma in pregnancy: a report of two cases and review of issues specific to the management of cervical carcinoma in pregnancy including planned delay of therapy. Cancer. 1998;82(8):1529-34. 
16. Marana HR, de Andrade JM, da Silva Mathes AC, Duarte G, da Cunha SP, Bighetti S. Chemotherapy in the treatment of locally advanced cervical cancer and pregnancy. Gynecol Oncol. 2001;80(2):272-4.

17. Caluwaerts S, Van Calsteren K, Mertens L, Lagae L, Moerman $P$, Hanssens $M$, et al. Neoadjuvant chemotherapy followed by radical hysterectomy for invasive cervical cancer diagnosed during pregnancy: report of a case and review of the literature. Int J Gynecol Cancer. 2006; 16(2):905-8.

18. Benhaim Y, Pautier P, Bensaid C, Lhommé C, Haie-Meder C, Morice P. Neoadjuvant chemotherapy for advanced stage cervical cancer in a pregnant patient: report of one case with rapid tumor progression. Eur J Obstet Gynecol Reprod Biol. 2008; 136(2):267-8.

19. Karam A, Feldman N, Holschneider $\mathrm{CH}$. Neoadjuvant cisplatin and radical cesarean hysterectomy for cervical cancer in pregnancy. Nat Clin Pract Oncol. 2007;4(6):375-80.

20. Bader AA, Petru E, Winter R. Long-term follow-up after neoadjuvant chemotherapy for high-risk cervical cancer during pregnancy. Gynecol Oncol. 2007; 105(1):269-72.

21. Palaia I, Pernice M, Graziano M, Bellati F, Panici PB. Neoadjuvant chemotherapy plus radical surgery in locally advanced cervical cancer during pregnancy: a case report. Am J Obstet Gynecol. 2007; 197(4):e5-6.

22. Boyd A, Cowie V, Gourley C. The use of cisplatin to treat advancedstage cervical cancer during pregnancy allows fetal development and prevents cancer progression: report of a case and review of the literature. Int J Gynecol Cancer. 2009; 19(2):273-6.

23. Seamon LG, Downey GO, Harrison CR, Doss B, Carlson JW. Neoadjuvant chemotherapy followed by post-partum chemoradiotherapy and chemoconsolidation for stage IIIB glassy cell cervical carcinoma during pregnancy. Gynecol Oncol. 2009; 1 14(3):540-1.

24. Favero G, Lanowska M, Schneider A, Marnitz S, Köhler C. Laparoscopic pelvic lymphadenectomy in a patient with cervical cancer stage $\mathrm{lb} l$ complicated by a twin pregnancy. J Minim Invasive Gynecol. 2010;17(1):118-20.

25. Chun KC, Kim DY, Kim JH, Kim YM, Kim YT, Nam JH. Neoadjuvant chemotherapy with paclitaxel plus platinum followed by radical surgery in early cervical cancer during pregnancy: three case reports. Jpn J Clin Oncol. 2010;40(7):694-8.

26. Rabaiotti E, Sigismondi C, Montoli S, Mangili G, Candiani M, Viganò R. Management of locally advanced cervical cancer in pregnancy: a case report. Tumori. 2010;96(4):623-6.

27. Weisz B, Schiff E, Lishner M. Cancer in pregnancy: maternal and fetal implications. Hum Reprod Update. $2001 ; 7(4): 384-93$.

28. Cardonick $E$, lacobucci $A$. Use of chemotherapy during human pregnancy. Lancet Oncol. 2004;5(5):283-91.

29. Marnitz S, Schmittel A, Bolbrinker J, Schmidt FP, Fons G, Kalache K, et al. The therapeutic management of a twin pregnancy complicated by the presence of cervical cancer, following laparoscopic staging and chemotherapy, with an emphasis on cisplatin concentrations in the fetomaternal compartments amnion fluid, umbilical cord, and maternal serum. Fertil Steril. 2009;92(5):1748.e 1-4.

30. Hadlock FP, Harrist RB, Martinez-Poyer J. In utero analysis of fetal growth: a sonographic weight standard. Radiology. 1991;181(1):129-33.

31. Van den Broek NR, Lopes AD, Ansink A, Monaghan JM. "Microinvasive" adenocarcinoma of the cervix implanting in an episiotomy scar. Gynecol Oncol. 1995;59(2):297-9. 